\title{
Comparative study of atomic force mode and tunneling mode tip-enhanced Raman spectroscopy
}

\author{
G. Picardi ${ }^{1, a}$, Q. Nguyen $^{1}$, J. Schreiber ${ }^{2}$, and R. Ossikovski ${ }^{1}$ \\ 1 LPICM, École Polytechnique, CNRS, 91128 Palaiseau, France \\ ${ }^{2}$ HORIBA Jobin Yvon SAS, Raman Division, 231 rue de Lille, 59650 Villeneuve d'Ascq, France
}

Received: 19 July 2007 / Accepted: 22 August 2007

Published online: 31 October 2007 - (C) EDP Sciences

\begin{abstract}
In Tip-Enhanced Raman Spectroscopy (TERS) a metal (or metallized) sharp tip is used to enhance the electromagnetic field by a localized surface-plasmon excitation. Two different modes - atomic force mode (AFM) and scanning tunneling mode (STM) - together with their respective types of probe tips are used in TERS experiments. We have compared the efficiency in enhancing the Raman signal on a thin dye layer for metal-coated AFM tips as well as for electrochemically etched metal STM tips. A much higher enhancement factor and better reproducibility were found when using STM tips. The very different performance is mainly attributed to the more efficient plasmonic excitation when using bulk-metal tips and possibly to the morphological differences in the tip and apex shapes existing between the two tip types.
\end{abstract}

PACS. 07.79.Fc Near-field scanning optical microscopes - 07.79.Lh Atomic force microscopes - 78.30.-j Infrared and Raman spectra - 73.20.Mf Collective excitations

\section{Introduction}

Raman spectroscopy, an optical characterization technique widely used for chemical and structural analysis, is intrinsically limited in spatial resolution to roughly half the wavelength of the excitation light, that is, in case of UV-Raman, to approximately $150 \mathrm{~nm}$ [1]. On the other side, scanning probe microscopies (SPM) are preferentially used for high resolution surface imaging, routinely showing the arrangements of individual molecules or atoms but lacking any direct chemical sensitivity.

Recently, Raman spectroscopy has been combined with either an atomic force microscope (AFM) [2-4] or a scanning tunneling microscope (STM) [5] in a novel technique called tip enhanced Raman spectroscopy (TERS) or scanning near-field Raman spectroscopy. TERS has the premises to perform optical imaging and spectroscopic analysis with sub-diffraction limited resolution. Within this approach, a nanometer-sized metal ( $\mathrm{Au}$ or $\mathrm{Ag}$ ) object, i.e. the end apex of a probe tip, is placed inside the laser spot. By acting as a local antenna, the tip enhances by several orders of magnitude the incident electromagnetic field in its close surrounding. Only molecules located within the near field of the enhancing tip are affected and thus probed by TERS. The field enhancement stems from a combination of the electrostatic lightning rod effect and the excitation of Localized Surface Plasmons (LSP) at the tip apex. TERS has potentially a wide range of applications: from materials nano-characterization in the semiconductor industry to single molecule studies in the biological field. A

\footnotetext{
a e-mail: gennaro.picardi@polytechnique.edu
}

TERS enhancement of up to $10^{6}$ (enough to attain single molecule sensitivity) was reported for a dye layer between a gold tip and a flat gold surface [6,7], while a $14-\mathrm{nm}$ lateral resolution has been reached in Raman imaging of single wall carbon nanotubes $[8,9]$.

Several factors affect the enhancement observed in the experiments, such as the tip material, the tip shape, the nature of the sample substrate underneath the tip, the illumination configuration and the tip-sample distance. In this paper, we present an experimental TERS study comparing etched metal tips used in STM mode and metalcoated tips used in AFM contact mode. The comparison was performed on thin dye layer samples deposited on a metal surface. In Section 2 the experimental setup, as well as the tip and sample preparation procedures, are described. The TERS experimental results are presented in Section 3 and discussed in Section 4, by analyzing critically the possible factors influencing the observed signal enhancement, such as tip material and geometry.

\section{Experimental}

A high resolution Raman spectrometer (HR800 from HORIBA Jobin Yvon) is coupled to an AFM microscope (XE-100 from PSIA) through a home-made optomechanical coupling system (Fig. 1). The system consists of three mirrors, four manual translational stages and a long working-distance objective $(50 \times, \mathrm{NA}=0.45$ from Olympus) oriented at $70^{\circ}$ with respect to the sample normal. The same objective is used for backscattering 


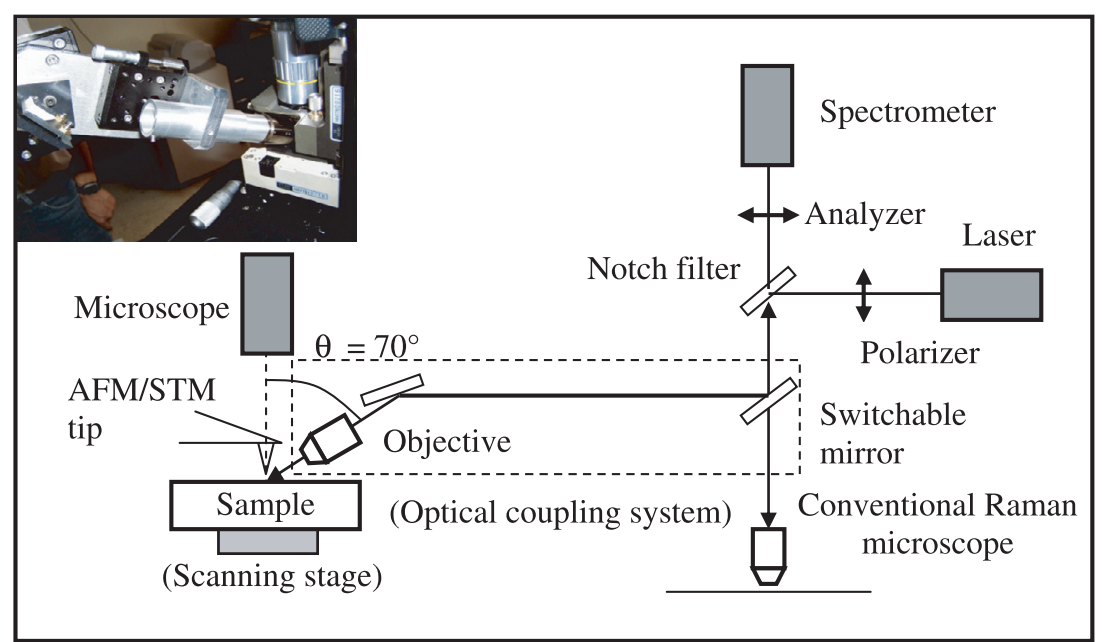

Fig. 1. Schematics of the TERS set-up. A picture of the $70^{\circ}$-tilted Raman objective and the AFM microscope sample holder is given in the insert.

collection of the Raman radiation. The XE-100 head has been modified (see insert in Fig. 1) in order to allow for the close approach of the objective to the tip-sample region. The system supports both AFM and STM operating modes by changing the corresponding tip support. A 633 -nm-wavelength He-Ne laser beam ( $p$-polarization) is focused to the tip apex and aligned through the micrometer stages. Laser power was kept below $1 \mathrm{~mW}$ in order to avoid sample heating and possible tip apex deterioration.

Commercially available AFM (from Veeco, Mikromasch, Nanosensors) tips were coated by thermal evaporation with a 10-nm adhesive $\mathrm{Cr}$ layer followed by a 20to $40-\mathrm{nm} \mathrm{Au}$ coating (evaporation speed: $0.2-0.1 \mathrm{~nm} / \mathrm{s}$ ). STM tips were prepared by electrochemical etching of a $0.25-\mathrm{mm} \mathrm{Au}$ wire (from Goodfellow) in a concentrated $\mathrm{HCl}$ /ethanol 1:1 mixture. Tips with final apex radii lower than $30 \mathrm{~nm}$ can be reproducibly prepared in this way $[10$, 11]. A home made circuit automatically switches off the etching current as soon as the lower portion of the wire detaches. Figures $2 \mathrm{a}$ and $2 \mathrm{~b}$ show scanning electron microscope (SEM) pictures of an AFM and an STM tip, respectively.

A dye layer was deposited on an Au-coated substrate by casting a single drop from a $10^{-6} \mathrm{M}$ Brilliant Cresyl Blue (BCB) solution in ethanol and subsequent rinsing in the pure solvent in order to remove any excess material and to have only a molecular layer adsorbed on the metal surface. The Au-coated substrate is essential for fluorescence quenching and for the tunneling feedback control in the STM operating mode. Flame-annealed atomically smooth $\mathrm{Au}(111)$ surfaces (from Arrandee) and a 60-nm evaporated $\mathrm{Au}$ layer on glass were used, for STM and AFM mode measurements, respectively.

In each experiment, two Raman spectra were acquired in the following sequence: the tip was first approached to the sample (in STM current-feedback mode or in AFM contact mode with, respectively, $1-0.5 \mathrm{nA}$ tunneling current at $0.1 \mathrm{~V}$ and $1-5 \mathrm{nN}$ contact force) and the laser spot was focused onto the tip - sample contact point with the help of the oblique view from the spectrometer camera and the translational stages. A "tip-enhanced" Raman spectrum was then collected with the tip engaged. The tip was subsequently retracted at a distance of several $\mu \mathrm{m}$ while maintaining the laser alignment and a second spectrum was recorded. The above procedure may be repeated several times on the same or on another sample point as a reproducibility check.

\section{Results}

In a TERS experiment, the observed contrast $C$ is defined as the ratio of the Raman scattered intensity (for a specific band) with the tip engaged and the tip withdrawn:

$$
C=I_{\text {tip engaged }} / I_{\text {tip withdrawn }} \text {. }
$$

This ratio can be used as a measure of the ability of a TERS experiment to discriminate the contribution to the signal due to the tip enhancement from that coming from the entire illuminated surface. Figures $3 \mathrm{a}$ and $3 \mathrm{~b}$ show typical Raman spectra of the BCB dye layer with and without the presence of the tip, in AFM and STM modes respectively. A maximum contrast of $1.4\left(\right.$ at $\left.585 \mathrm{~cm}^{-1}\right)$ was found in AFM mode while contrast values ranging from 20 up to 100 (depending on the tip-to-tip performance variation) were observed in STM mode. (The steep background increase observed in the "red" region of the Figure 3a spectrum is due to a stray light contribution from the red diode laser used for the AFM mode optical feedback.)

Because of the substantially lower contrast values obtained in AFM mode compared to STM mode ones, the tip-withdrawn-spectrum response in the AFM experiments was intentionally "boosted" by using an evaporated Au substrate presenting a granular roughness (about $2 \mathrm{~nm} \mathrm{rms}$ ) and consequently supporting some surface enhanced Raman (SERS) effect. By using a "mildly" activated SERS substrate it is thus possible to make visible even a very small signal enhancement due to the tip, 

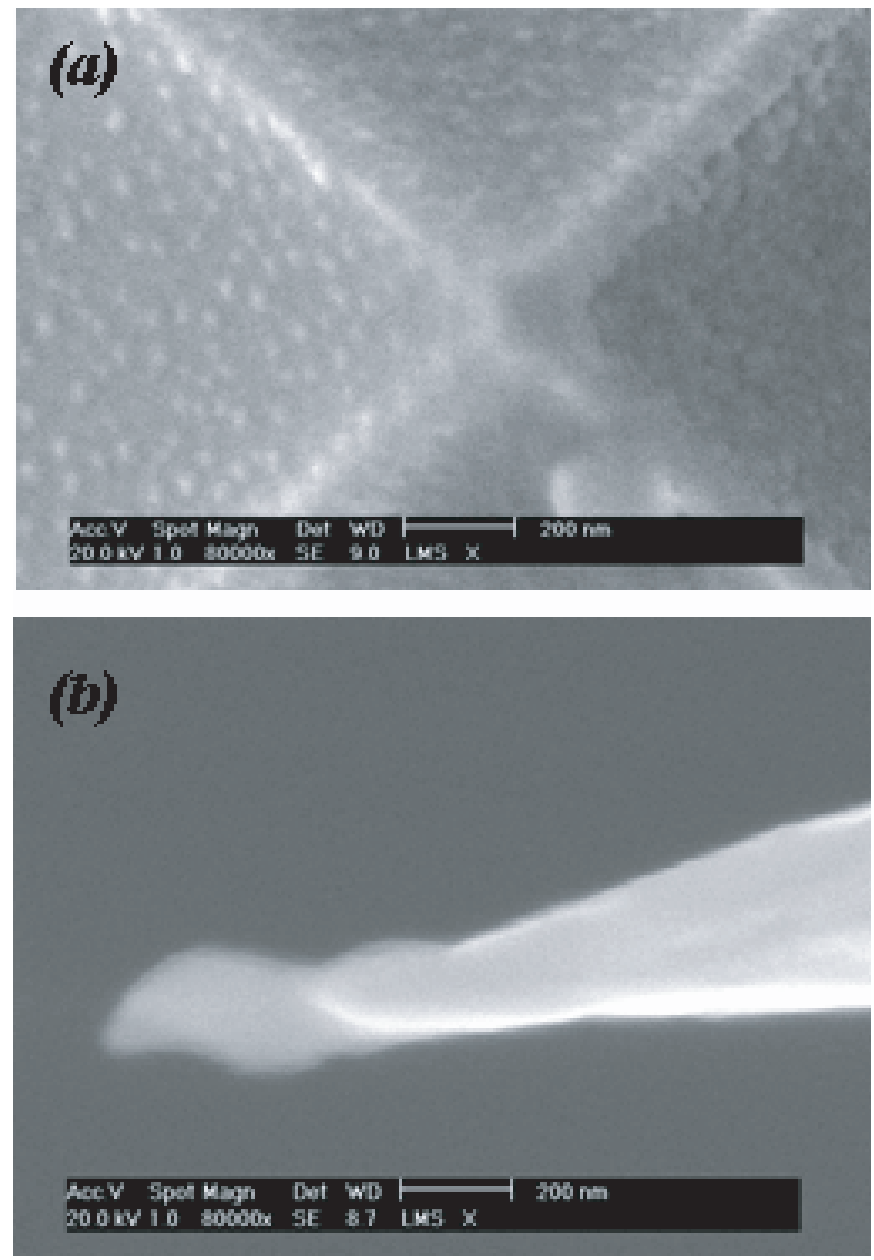

Fig. 2. SEM pictures of (a) an Au-coated AFM tip (top view) and (b) an etched-Au wire STM tip (side view). The flake at the STM tip apex results from carbon contamination from the vacuum pump.

as is the case in Figure 3b. In contrast to the AFM tip experiments, in the experiments with the STM etched tips the atomically smooth Au surface supports almost no SERS effect and the "tip engaged" spectrum is practically flat while the "tip-enhanced" spectrum is still remarkably high, see Figure 3b. AFM tips previously tested on these atomically smooth substrates were not able to provide any appreciable signal enhancement.

The difference in enhancement performance between STM and AFM tips is accompanied by a similar difference in enhancement reproducibility. TERS spectra recorded in STM mode showed quite different contrast values (owing to the tip-to-tip variation), but for almost all of the tested tips a clear Raman spectra exhibiting the dye vibrational fingerprints was recorded in tip-engaged position. On the contrary, despite testing different kinds of commercial AFM tips as well as slightly varying the evaporation procedure conditions (metal film thickness, evaporation speed, angle of the cantilever with respect to the crucible), a signal enhancement was observed only for a small number of AFM tips (about 20 percent).
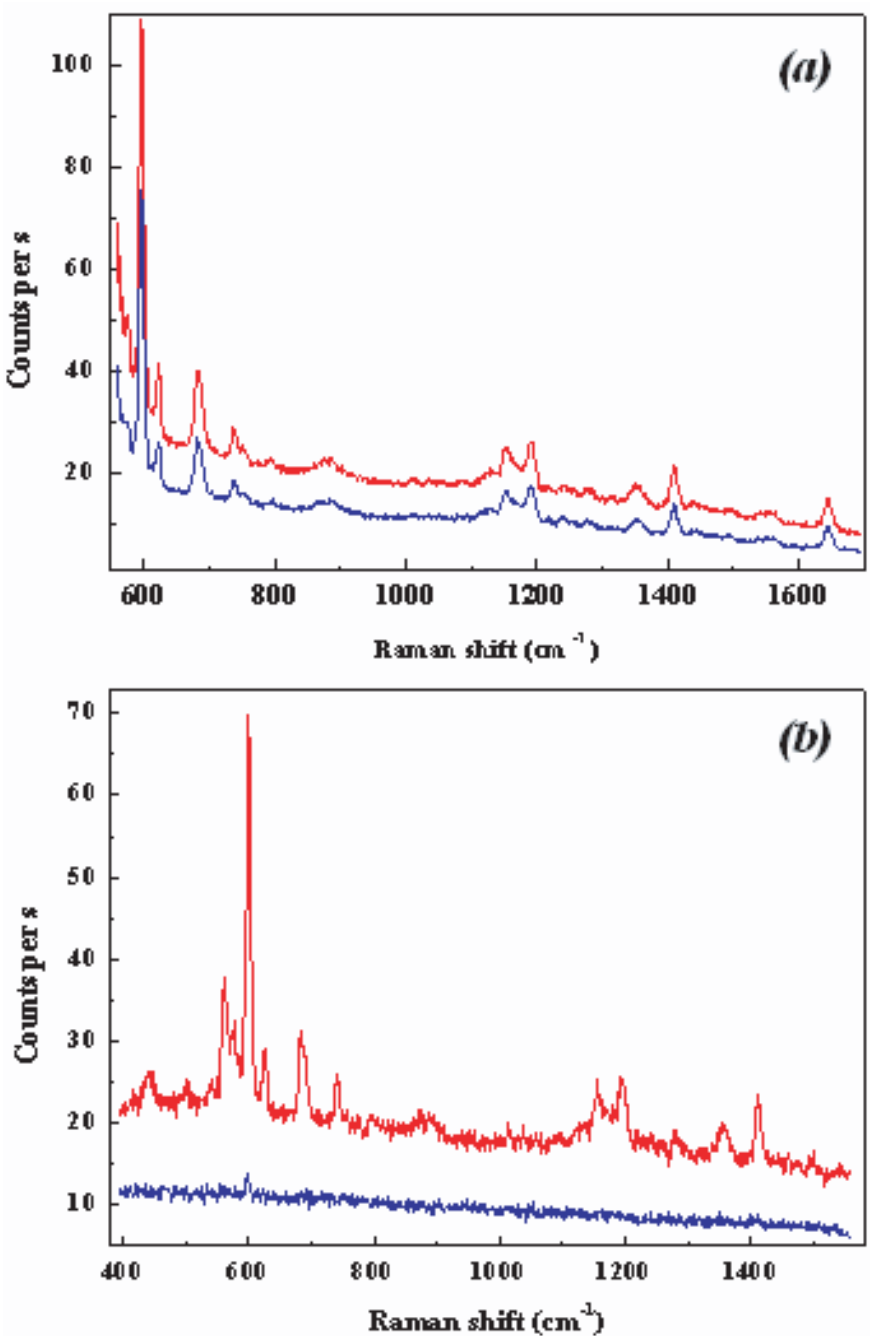

Fig. 3. Tip enhanced Raman spectra of a BCB dye layer deposited on metal surface recorded in AFM and STM modes. Lower trace corresponds to the tip withdrawn, the upper trace - to the tip engaged. (a) Au-coated AFM tip operating on a dye-covered evaporated $\mathrm{Au}$ film. Acquisition time: $10 \mathrm{~s}$. Laser power: $0.5 \mathrm{~mW}$. (b) Etched-Au STM tip operating on a dyecovered atomically smooth $\mathrm{Au}$ film. Acquisition time: $20 \mathrm{~s}$. Laser power: $0.5 \mathrm{~mW}$.

An approximate estimation of the Raman signal enhancement factor $(E F)$ can be performed by assuming that the difference in signal intensities between the "tip engaged" spectrum and the "tip withdrawn" spectrum is mainly due to those molecules of the sample that are closest to the tip apex since the enhanced (or near) field generated by the plasmon excitation at the tip apex decays very rapidly with moving away from the tip. Within the simplest configurational approximation, the tip enhanced signal comes from a circular area with radius comparable to the tip apex radius. In this case the enhanced field can be considered to be constant over a circle, although different and more realistic field distributions below the tip apex have been taken in account by Pettinger et al. [12]. Therefore, the $E F$ can be calculated from the experimental $C$ 
values by normalizing the signals by the corresponding laser illuminated (or focal) area $A$ and by the TERS circular area

$$
C=1+E F\left(S_{\text {tip engaged }} / S_{\text {tip withdrawn }}\right)=1+E F\left(\pi r_{\text {tip }}^{2} / A\right) .
$$

As a direct consequence of the oblique incidence, the laser beam forms an elliptical spot on the sample with major axis $a=D / \cos 70^{\circ}$ and minor axis $b=D$, where $D$ is the diffraction-limited resolution $(D=0.61 \lambda / \mathrm{NA})$ estimated to be $\sim 860 \mathrm{~nm}$ for our setup. The resulting spot area $A$ is approximately $6-7 \mu \mathrm{m}^{2}$. The tip radius of curvature, as evaluated from SEM images, was $\sim 40 \mathrm{~nm}$ for the coated AFM tips and $\sim 30 \mathrm{~nm}$ for the STM tips. By taking into account these values we estimated a Raman signal $E F$ of $\sim 5 \times 10^{2}$ with AFM tips and of $4 \times 10^{4}$ to $2 \times 10^{5}$ with STM tips. These approximate values are very likely lower bound estimations since the enhanced signal possibly stems from a much smaller area (because of the tip-enhanced field likely peaking very sharply at the apex end point); nonetheless, metal tips perform much better than metal-coated ones and, in our observations, in a more reproducible way also. It is to be noted that similar observations were reported by other groups [13].

\section{Discussion}

The very different TERS performance of the metal or metal-coated tips we observed can be attributed to a number of causes. First of all, the main contribution to the local electric field (i.e., to the scattered Raman signal) enhancement is related to the excitation of LSP modes at the tip apex. Then, a first straightforward conclusion is that the plasmonic excitation at the AFM tip apexes, metalcoated by evaporation, is not very efficient. It has been experimentally evidenced that the substrate material onto which a thin gold film is evaporated strongly influences the LSP resonant frequencies [14]. In particular, a pronounced shift of the plasmon resonance wavelength $\lambda_{S P R}$ to the infrared is observed with increasing the dielectric constant of the tip material (commercial AFM tips are made of Si or $\mathrm{Si}_{3} \mathrm{~N}_{4}$ ). As a consequence, the resulting plasmon excitation may appear somewhat dampened in the visible range. On the contrary, no "substrate" dampening is expected to take place when working with bulk metal tips.

One more cause for the better results obtained with the STM etched tips can be advanced. It may be conjectured that the TERS effect is not generated by the entire tip apex seen on the SEM pictures (presenting a typical radius of $30-50 \mathrm{~nm}$ ) but is rather created by smaller nanoscale structure(s) (sizing from 5 to $10 \mathrm{~nm}$ ) distributed on the apex. In contrast, metal evaporation produces a relatively homogeneous albeit granular layer possibly supporting only "mild" plasmonic activity. On the other hand, the electrochemical etching procedure is known to be more efficient in generating higher roughness at the apex (e.g., double or multiple tips). Consequently, the STM apex shape can be only loosely approximated by a half sphere (or a half ellipsoid). A "nano-star" [15] appears as a more adequate description of the apex surface presenting a number of protusions that (cooperatively) support plasmon excitation.

Regarding other differences between the two kinds of tips, it can be readily found either from the SEM pictures or from the microscope side view that the STM tips present a smaller aperture angle $\left(<10^{\circ}\right)$ with respect to that of common AFM tips ( $35^{\circ}$ typically). The field buildup at a sharper protusion is stronger (lightning-rod effect), and thus it is generally preferable to employ sharper tips. Using a sharper (and conical) tip also reduces the amount of reflected light that may contribute to the observed signal increase without bringing any real electro-magnetic enhancement due to plasmon excitation or any real nearfield resolution; an extremely delicate issue when TERS probing bulk Raman-active samples [16]. Apart from the performance itself, the crucial laser alignment at the tipsample contact point is considerably easier with a tip having a sharper cone, since the sharpness of the microscope lateral view is not compromised by the unfocused light scattered by a more massive body, as a pyramidal or a blunt tip.

Finally, in the two modes, AFM and STM, one more difference may affect the experimental results namely the distance from the tip to the probed surface. In the STM case the tip is constantly held at $\sim 1 \mathrm{~nm}$ distance, forming a nano-cavity with the underlying metal. The theory of resonant plasmon modes confined in a gap was extensively used to model STM - light emission spectra from $\mathrm{Au}$ - air - $\mathrm{Au}$ (or other metal - insulator-metal) junctions [17-19]. Concisely, a tunneling junction supports additional ("gap") modes stretching in frequencies from the (cavity material) limiting surface plasmon frequency to the infrared.

In this context, we also point out at the importance of the substrate. An ideal optical coupling between the tip and the substrate is obtained when both are responsive (in resonant condition) to the exciting radiation. The polariton resonances localized inside the $\mathrm{Au}(\mathrm{Ag})-\mathrm{Au}(\mathrm{Ag})$ cavity are strongly excited by the red (green) light and generate a much stronger and localized field than the one that is available at the tip (or any other isolated geometrical structure) alone [20]. The morphology of the sample surface may also modulate the TERS signal: with lower signals recorded (from molecules adsorbed) on a flat terrace and higher signal when the tip is on a small hillock or other surface defect, or even on single steps [21].

The main advantage of using metal-coated AFM tips consists in their relatively simple preparation and commercial availability as well as on the absence of any restriction on the nature (conducting or not) of the sample to be characterized. On the other side, tips prepared by electrochemical etching are by far less expensive and their fabrication is likewise easy and more reliable; however, when used with a tunneling feedback in STM mode a strong limitation exists due to the required conductive nature of the substrate [22]. A less limiting approach consists in using the etched tips as glued to a tuning fork operated 
in shear-force feedback mode [23,24]. Different tip-coating procedures exist [25] and the possibility to further refine the tip shape by ion milling can also be considered. However, these preparation methods become less straigthforward and, once again, the general reproducibility has still to be demonstrated.

\section{Conclusions}

We presented a comparative study of two commonly used TERS experimental approaches: using metal-coated tips in AFM contact mode and using etched-metal tips in STM mode. The experimental results on a dye layer deposited on a metal film indicate a much stronger TERS effect and overall better reproducibility when using metal-etched STM tips. Possible reasons for this observation, such as the intrinsically lower "optical quality" of the coated tips with respect to the massive metal ones as well as the differences in tip shapes, have been discussed. We believe that this study may prove helpful when planning a TERS experiment.

One of the authors (Q. Nguyen) gratefully recognizes a CIFRE PhD scholarship by Ecole Polytechnique and HORIBA Jobin Yvon.

\section{References}

1. V. Poborchii, T. Tada, T. Kanayama, Appl. Phys. Lett. 89, 233505 (2006)

2. R.M. Stoeckle, Y.D. Suh, V. Deckert, R. Zenobi, Chem. Phys. Lett. 318, 131 (2000)

3. M.S. Anderson, Appl. Phys. Lett. 76, 3130 (2000)

4. N. Hayazawa, A. Tarun, Y. Inouye, S. Kawata, J. Appl. Phys. 92, 6983 (2002)

5. B. Pettinger, G. Picardi, R. Schuster, G. Ertl, Single Mol. 3, 285 (2002)
6. B. Pettinger, B. Ren, G. Picardi, R. Schuster, G. Ertl, Phys. Rev. Lett. 92, 096101 (2004)

7. K.F. Domke, D. Zhang, B. Pettinger, J. Am. Chem. Soc. 128, $14721(2006)$

8. A. Hartschuh, E.J. Sanchez, X. Sunney Xie, L. Novotny, Phys. Rev. Lett. 90, 095503 (2003)

9. N. Anderson, A. Hartschuh, S. Cronin, L. Novotny, J. Am. Chem. Soc. 127, 2533 (2005)

10. B. Ren, G. Picardi, B. Pettinger, Rev. Sci. Instrum. 75, 837 (2004)

11. L. Billot, L. Berguiga, M. Lamy de la Chapelle, Y. Gilbert, R. Bachelot, Eur. Phys. J. Appl. Phys. 31, 139 (2005)

12. B. Pettinger, B. Ren, G. Picardi, R. Schuster, G. Ertl, J. Raman Spectrosc. 36, 541 (2005)

13. T. Schmid, T.A. Schmitz, P.D. Setz, B.S. Yeo, W. Zhang, R. Zenobi, Chimia 60, (2006)

14. B.S. Yeo, T. Schmid, W. Zhang, R. Zenobi, Anal. Bioanal. Chem. 387, 2655 (2007)

15. F. Hao, C.L. Nehl, J.H. Hafner, P. Nordlander, Nano Lett. 7, $729(2007)$

16. C. Georgi, M. Hecker, E. Zschech, Appl. Phys. Lett. 90, 171102 (2007)

17. R.W. Rendell, D.J. Scalapino, Phys. Rev. B 24, 3276 (1981)

18. J.A. Porto, P. Johansson, S.P. Apell, T.L. Lopez-Rios, Phys. Rev. B 67, 085409 (2003)

19. K. Meguro, K. Sakamoto, R. Arafune, M. Satoh, S. Ushioda, Phys. Rev. B 65, 165405 (2002)

20. A. Cvitkovic, N. Ocelic, J. Aizpurua, R. Guckenberger, R. Hillenbrand, Phys. Rev. Lett. 97, 060801 (2006)

21. W. Zhang, X. Cui, B.S. Yeo, T. Schmid, C. Hafner, R. Zenobi, Nano Lett. 7, 1401 (2007)

22. It is to be stressed again that better TERS performance is observed on metallic substrates whatever the operating mode (AFM or STM)

23. C.C. Neacsu, J. Dreyer, N. Behr, M.B. Raschke, Phys. Rev. B 73, 193406 (2006)

24. N. Anderson, A. Hartschuh, L. Novotny, Nano Lett. 7, 577 (2007)

25. J.J. Wang, Y. Saito, D.N. Batchelder, J. Kirkham, C. Robinson, D.A. Smith, Appl. Phys. Lett. 86, 263111 (2005) 